\title{
New Audiences for Ontologies: Dealing with Complexity in Business Processes
}

\author{
Jeroen van Grondelle \\ Be Informed, The Netherlands \\ j.vangrondelle@beinformed.com \\ http://www.beinformed.com/
}

Today, governments and businesses have to deal with high degrees of complexity: The products they offer are highly individualized, there are many regulations they must comply with and all this has to be dealt with under a growing rate of change. Many organizations have tried to meet this challenge by reducing complexity, through the elimination of exceptions etc. Jeroen van Grondelle, principal architect at Be Informed, argues that the only way to deal with complexity is by embracing it.

Ontologies have proven to be an excellent way to deal with all the different concepts that are introduced when products are defined and the supporting business processes are designed. When the right conceptualization is chosen, ontologies capture these policy choices and constraints in a natural way. Ontologies deal well with the heterogeneous nature of policy and regulations, which often originate from different legal sources and have different owners.

The benefits exist throughout the entire policy lifecycle. The formal, precise nature of ontologies improves the quality and consistency of the choices made and reduces ambiguity. Because ontologies are well interpretable by machines, Be Informed succeeds in inferring many of the supporting services, such as process applications and decision services, from the ontologies, thereby eliminating the need for systems development. And the ability to infer executable services also allows for advanced what-if analysis and simulation of candidate policies before they are put into effect.

Jeroen will show some examples where ontologies were successfully applied in the public sector and what the impact was on all parties involved, from policy officers to citizens. He will also present some of the research challenges encountered when these new audiences are confronted with ontologies, a technology that typically is of course unfamiliar to them. 